RESEARCH REPORT

\title{
Type 2 diabetes in grandparents and birth weight in offspring and grandchildren in the ALSPAC study
}

\author{
P McCarron, G Davey Smith, A T Hattersley, and ALSPAC Study Team
}

J Epidemiol Community Health 2004;58:517-522. doi: 10.1136/jech.2003.007989

See end of article for authors' affiliations

.....................

Correspondence to:

Dr P McCarron,

Department of

Epidemiology and Public

Health, Queen's University

Belfast, Mulhouse Building,

Grosvenor Road, Belfast

BT12 6BJ, UK; peter.

mccarron@qub.ac.uk

Accepted for publication 28 August 2003

\begin{abstract}
Objective: To examine the association between a history of type 2 diabetes and birth weight of offspring and grandchildren.

Design: Prospective observational study. Diabetic status, as reported by mothers (F1 generation) was collected on grandparents (FO) of babies (F2) born to mothers (F1) who participated in a study of maternal and child health. Associations between risk of grandparental diabetes and birth weight in mothers (F1) and grandchildren (F2) were analysed using linear and logistic regression.

Setting: Avon: comprising of the city of Bristol and surrounding areas.

Participants: 12076 singleton babies (F2), their parents (F1) and maternal and paternal grandparents (FO).

Results: Women (F1) who had no parents with type 2 diabetes had lower birth weights than women with one or two diabetic parents, after controlling for the age of both parents. There was a $U$ shaped association between maternal birth weight and grandmaternal diabetes, but no evidence of an association with grandpaternal diabetes. The grandchildren of maternal grandparents with type 2 diabetes were more likely to be in the top tertile of birth weight than grandchildren of non-diabetics. There was evidence for an inverted $U$ shaped association between birth weight of grandchildren and diabetes in paternal grandmothers.

Conclusions: This is the first study to show intergenerational associations between type 2 diabetes in one generation and birth weight in the subsequent two generations. While the study has limitations mainly because of missing data, the findings nevertheless provide some support for the role of developmental intrauterine effects and genetically determined insulin resistance in impaired insulin mediated growth in the fetus.
\end{abstract}

S tudies investigating the role of early life factors in the development of chronic disease have demonstrated both $\mathrm{U}$ shaped $^{1}$ and inverse ${ }^{2}$ associations of birth weight with insulin resistance and type 2 diabetes in later life. Associations with high birth weight may be attributable to a high prevalence of maternal type 2 diabetes, ${ }^{13}$ or gestational diabetes, ${ }^{4}$ while it has been hypothesised that low birth weight associations are attributable to a programmed response to fetal undernutrition, ${ }^{56}$ or to genetically determined insulin resistance causing both low insulin mediated fetal growth and subsequent insulin resistance in adulthood-the "fetal insulin hypothesis".

The positive associations between parental and offspring birth weight ${ }^{8-11}$ suggest that there may in turn be associations between chronic disease in parents-and possibly earlier generations-and offspring fetal growth. Evidence for such birth weight intergenerational effects has come from both animal and human studies. Pregnant rats fed a protein deficient diet over 12 generations had fetuses in which growth became poorer in each subsequent generation, and after restoration of a normal diet a further three generations were required before growth was normalised. ${ }^{12}$

Studies in human populations have reported inverse associations between birth weight and parental cardiovascular disease mortality ${ }^{13-15}$ and maternal blood pressure ${ }^{16}$ and several research groups have now also shown that birth weight of offspring is inversely related to risk of diabetes in parents. An inverse association between birth weight of offspring and risk of type 2 diabetes in fathers was demonstrated among Pima Indians, a population with a high prevalence of this disorder. ${ }^{17}$ More recently, studies in populations with lower diabetes prevalence have reported that birth weight of offspring is inversely related to insulin resistance in 60-79 year old women, ${ }^{18}$ that birth weight adjusted for gestational age, is inversely related to both maternal and paternal diabetic mortality, ${ }^{19}$ and that reported maternal and paternal type 2 diabetes is associated with lower offspring birth weight in the 1958 birth cohort. ${ }^{20}$ These findings point to the contribution of common genetic factors to the relation between birth weight and risk of type 2 diabetes.

The Avon longitudinal study of parents and children (ALSPAC), formerly known as the Avon longitudinal study of pregnancy and childhood, afforded an opportunity to further explore such intergenerational effects. The following hypotheses were tested:

- Grandmothers (F0 generation) who develop type 2 diabetes in later life have both (a) a higher prevalence of large babies (F1) than non-diabetic grandmothers (reflecting the well known effects of frank diabetes and gestational diabetes on birth weight), and (b) maternal diabetes genes resulting in low birth weight babies;

- Grandfathers (F0) with type 2 diabetes have, through diabetes genes, smaller offspring (Fl) than non-diabetic grandfathers;

- Daughters (F1) of diabetic parents (F0) have, like their mothers, both heavy and fetal growth restricted babies (F2).

\section{METHODS}

ALSPAC is a prospective observational study of pregnant women resident in Avon in the south west region of England 
who had an expected date of delivery between 1 April 1991 and 31 December 1992. ${ }^{21}$ The cohort, which is broadly representative of the UK population in terms of socioeconomic position, has been described in detail elsewhere. ${ }^{22}$ Briefly, the overall aim of the study was to determine environmental and genetic factors associated with the health and development of the fetus and child. Detailed information was obtained from the mothers (Fl) via self completion questionnaires administered during pregnancy, and birth details of the babies (F2) were added. Data collected included:

- Maternal and paternal grandparents (F0): age (years), cigarette smoking (yes, no), diabetes not requiring insulin as reported by offspring (yes, no), subsequently labelled "type 2 diabetes";

- Mother (F1): birth weight (g), height $(\mathrm{cm})$, parity $(0,1+)$, ethnicity (white, other), cigarette smoking during pregnancy (yes, no);

- Baby (F2): sex, birth weight (g), gestational age (weeks).

People with missing data on the above variables were excluded from the analyses, and parents with reported birth weights over $5640 \mathrm{~g}$ (this being the highest birth weight in the F2 generation) were also omitted.

Figure 1 shows the three generations and the hypothesised associations of subsequent type 2 diabetes risk.

\section{STATISTICAL ANALYSES}

Type 2 diabetes in maternal grandparents (FO) and birth weight of mothers (F 1 )

Linear regression was used to compute birth weights of mothers, adjusted for grandparental age, according to grandparental type 2 diabetic status: (a) neither maternal grandparent, (b) maternal grandmother only, (c) maternal grandfather only, or (d) both grandparents having diabetes. Logistic regression was used to calculate odds ratios (ORs) for the association between grandparental type 2 diabetes and mother's birth weight. Crude analyses, and analyses controlling for age and smoking status of grandparents, were performed. To search for a non-linear relation likelihood ratio tests were carried out with and without the square of maternal birth weight.

\section{Type 2 diabetes in grandparents (FO) and birth weight} of grandchildren (F2)

Linear regression analyses were used to test for differences between fully adjusted mean birth weights (adjusted for sex and gestational age) of grandchildren according to grandparental type 2 diabetic status: (a) at least one maternal grandparent only, (b) at least one paternal grandparent only, (c) at least one maternal and at least one paternal grandparent, and (d) neither grandparent having diabetes. ORs were calculated in a similar way to those for mothers (F1), first using birth weight adjusted for gestational age and sex of the baby, with further adjustment for the square of gestational age, mother's height, smoking status of mother during pregnancy, parity, and ethnic origin, and additionally controlling for age and smoking status of the grandparent. Because of missing data, particularly in the Fo generation, we also examined the birth weight distributions of the F2 generation for babies by availability of grandparents age and diabetic status. We further examined the association between grandparental type 2 diabetes risk and availability of data by age of grandparents. All analyses were performed using Stata 7.0 (Stata, College Station, TX).

\section{RESULTS}

Birth weights were available for 6978 (57.8\%) mothers (F1). After omitting one mother with an improbable birth weight the mean birth weight (standard deviation) was 3263 (607) g. For the 12076 babies (F2) (5861 girls and 6215 boys) available for inclusion in the analyses, mean birth weight of girls was 3368 (510) g, and that of boys was 3476 (559) g. Babies for whom grandparental age data were available were $20.6 \mathrm{~g}$ to $101.8 \mathrm{~g}$ heavier at birth and babies for whom grandparental diabetic status data were available were $24.4 \mathrm{~g}$ to $109.0 \mathrm{~g}$ heavier than babies for whom these data were not available. Mean gestational age was 39.5 (1.8) weeks. The correlation between infant (F2) birth weight and maternal (Fl) birth weight was 0.23 $(\mathrm{p}<0.001)$.

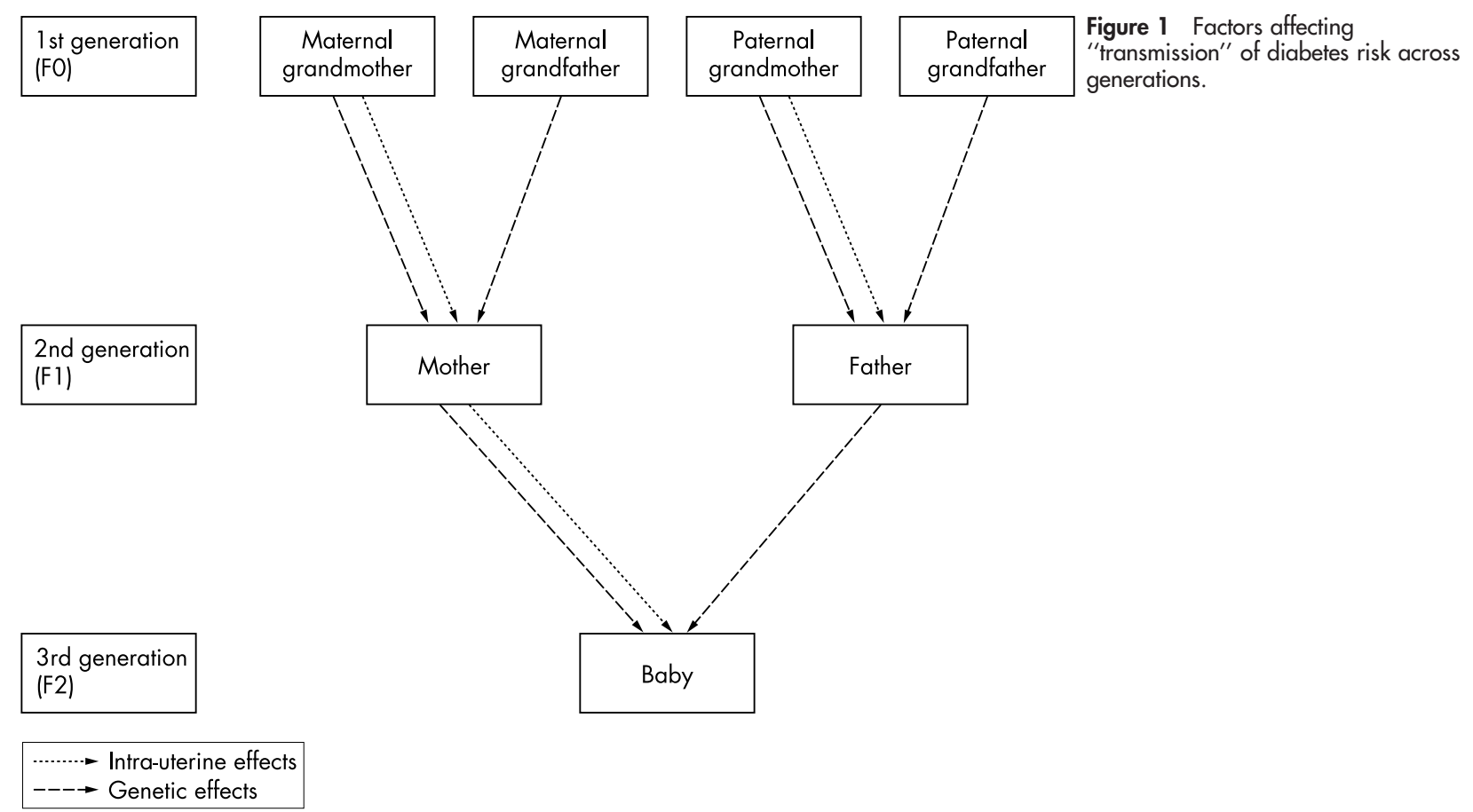




\section{Key points}

- Intergenerational associations exist between type 2 diabetes in one generation and birth weight in the subsequent two generations.

- Both intrauterine environmental factors and genetically determined insulin resistance may contribute to impaired insulin mediated growth in the fetus.

- These intergenerational effects are seen in a population without a very high prevalence of type 2 diabetes.

- Replication of these investigations in studies with both data on early life growth and DNA on ancestors and offspring could improve understanding of diabetes aetiology.

\section{Type 2 diabetes in grandparents (FO) and birth weight of mothers (F1)}

Table 1 shows the completeness of reporting of type 2 diabetic status of grandparents (F0) and the numbers and proportions for whom offspring (F1) birth weights were available. Mothers and fathers with available birth weight data had the same probability of having a diabetic parent as non-reporters. Although there were statistically significant differences in the parity, ethnic background, the proportion of mothers who smoked during pregnancy, height, and infant birth weight of reporters compared with non-reporters, these differences were very small (data not shown). Data on diabetic status were available on over $93 \%$ of maternal grandparents compared with over $65 \%$ of paternal grandparents. The prevalence of diabetes was similar for both sets of grandparents. Data on parental (F1) birth weights were available for just under $60 \%$ and $25 \%$ of mothers and fathers respectively. In view of the paucity of data on the diabetic status of paternal grandparents (F0) and the birth weight of fathers (Fl), analyses of the association between type 2 diabetes in grandparents and birth weight of their own offspring were restricted to investigation of the association in maternal grandparents (F0) and their daughters (Fl).

Table 2 shows the birth weights of mothers, adjusted for grandparental age, according to grandparental diabetic status. These measures provide some evidence that women whose mother, father, or both parents (in the last case based on only five mothers) had type 2 diabetes were heavier at birth than women whose parents did not have diabetes.

Table 3 gives ORs for the associations of grandparental type 2 diabetes and mother's birth weight. As unadjusted analyses for the full cohort were very similar to those for individuals with data available on potential confounders, we report only the latter here. There is evidence for a $U$ shaped association between type 2 diabetes in maternal grandmothers (adjusted for age) and maternal birth weight that remained after controlling for grandmother's educational status and smoking history. However, there was no association between type 2 diabetes in maternal grandfathers and birth weight of mothers.

\section{Type 2 diabetes in grandparents (FO) and birth weight of grandchildren (F2)}

From linear regression analyses, infant birth weight, adjusted for sex and gestational age of the baby provide some evidence that babies who had at least one maternal grandparent with type 2 diabetes were heavier than those babies who had at least one paternal grandparent or no diabetic grandparents (table 4).

Table 5 shows ORs for the association between grandparental type 2 diabetes and tertile of infant birth weight. Despite the reduction in the number of people available for analysis, because of lack of confounder data on the F0 generation, there was little difference between the unadjusted results for the full and the reduced cohorts, and we therefore report only the latter here. There was a positive association between birth weight adjusted for sex and gestational age and type 2 diabetes in maternal grandmothers and also in maternal grandfathers. There was very little change when using fully adjusted birth weight and additionally adjusting for age and smoking history of maternal grandmother or grandfather as appropriate.

The inverse association between tertile of birth weight, adjusted for sex and gestational age, and type 2 diabetes in paternal grandmothers demonstrated evidence of nonlinearity. There was an inverted $U$ shaped association between grandchild's birth weight adjusted for sex and gestational age, and type 2 diabetes in paternal grandfathers but after further adjustment for other confounders the evidence for this association was lessened.

\section{DISCUSSION}

To our knowledge this is the first study to show intergenerational associations between type 2 diabetes in one generation and birth weight in the subsequent two generations in a population without a very high prevalence of type 2 diabetes. The findings offer some support for the role of both intrauterine prenatal development and parental genetic factors in establishing an association between type 2 diabetes in one generation and birth weight in the subsequent generations.

\section{Strengths and limitations of the study}

The major strength of the study is its large size and the wealth of data collected on three generations of individuals. This enabled us to compute a robust measure of fetal

Table 1 Number (\%) of grandparents (FO) with reported type 2 diabetes status and number (\%) of parents (F1) with reported birth weight

\begin{tabular}{lllll}
\hline & Maternal grandmother & Maternal grandfather & Paternal grandmother & Paternal grandfather \\
\hline $\begin{array}{l}\text { Number (\%)* grandparents with diabetes } \\
\text { status available }\end{array}$ & $11295(93.5)$ & $11269(93.3)$ & $7915(65.5)$ & $7915(65.5)$ \\
$\begin{array}{l}\text { Diabetes present (in those with reported } \\
\text { diabetic status) }\end{array}$ & & & & \\
Yes, Number (\%) & $232(2.1)$ & $303(2.7)$ & $143(1.8)$ & $215(2.7)$ \\
Number (\%) with parent birth weight & $142(2.0)$ & $176(2.5)$ & $48(1.6)$ & $75(2.6)$ \\
No, number (\%) & $11063(97.9)$ & $10966(97.3)$ & $7772(98.2)$ & $7700(97.3)$ \\
Number (\%) with parent birth weight & $6836(98.0)$ & $6802(97.5)$ & $2870(98.4)$ & $2843(97.4)$ \\
Number (\%) parents with reported birth & Mothers: 6978 (57.8) & & & \\
weight & & & & \\
\hline *From 12076 singleton births; tof parents with reported birth weight. & & & \\
\hline
\end{tabular}


Table 2 Association between diabetic status in maternal grandparents (FO) and birth weight of mothers (F1)

\begin{tabular}{ll}
\hline Maternal grandparents with diabetes $(\mathbf{n})$ & *Adjusted mean birth weight $(\mathbf{g})$ of mother $(95 \% \mathrm{Cl})$ \\
\hline Neither maternal grandparent (4748) & $3276(3275$ to 3278$)$ \\
Maternal grandmother (90) & $3487(3477$ to 3496$)$ \\
Maternal grandfather (123) & $3297(3289$ to 3305$)$ \\
Both maternal grandparents (5) & 3352 (3298 to 3407) \\
\hline
\end{tabular}

*Birth weight to nearest gram adjusted for age of maternal grandparents.

growth-adjusted birth weight-rather than having to use the relatively crude measure of unadjusted birth weight, and also allowed us to control for several important confounding variables in the analyses. As just over $1 \%$ of the study children had a sibling in the study, this small number would not have influenced the effect estimates or confidence intervals presented.

Several limitations deserve consideration. Most importantly, complete data were not available for all grandparents (F0) and parents (F1). Diabetic status was not available on over one third of paternal grandparents. Furthermore, type 2 diabetic status was assigned based on the mothers' (F1) report of whether grandparents had diabetes not requiring insulin. This will have missed some people with type 2 diabetes, as insulin is also used in the treatment of this condition. However, the proportions of maternal grandparents with type 2 diabetes as reported by mothers (F1) are in close agreement with published type 2 diabetes prevalence data for adults, including a self reported figure of $2.4 \%{ }^{23-26}$ The paternal grandparent diabetes data, although only available for a smaller proportion of these people, are also similar to published figures. The mean birth weight of the F2 babies included in the analyses was higher than that of those excluded because of missing data. However, there were only small differences between the results for the complete and the reduced datasets. This volume of missing data highlights the need for replication of the analyses in studies with more complete data on earlier generations.

Only a crude measure of gestational age was available on parents, and birth weight itself was not available on over $40 \%$ of mothers (F1). However, the broad similarity in the proportion of mothers with a diabetic parent and the proportion who smoked during pregnancy, and in parity, ethnic background, height, and infant birth weight, for women who reported their birth weight and those who did not, again indicates that significant bias is unlikely to be operating. Nevertheless, the data are somewhat limited and caution is needed in interpreting the results. As stated in the introduction, birth weight data were only available on a relatively low proportion of the Fl fathers, and therefore examination of the association between paternal birth weight and paternal grandparental risk of diabetes was not carried out. Also, as data on parents (F1) and grandparents (F0) were reported by the mother of the baby (F2) misclassification is possible, although several reports have shown that in women, self reported birth weight ${ }^{27}{ }^{28}$ and diabetic status ${ }^{3}$ are reliable. Finally, examination of the proportion of grandparents with type 2 diabetes among those mothers with and without reported birth weight also indicates that the likelihood of serious selection bias is low.

\section{Explanations of findings}

The observed associations between infant fetal growth or parental birth weight and grandparental risk of type 2 diabetes could reflect four basic processes. Firstly, socioenvironmental factors could influence the risk of type 2 diabetes in grandparents (F0) and the fetal growth of their descendants. This would generate similar associations between birth weight of parents (F1) and grandchildren (F2), and type 2 diabetes risk in grandmothers and grandfathers, but this was not the case in this study.

Secondly, shared environmental triggers of diabetes in later life could also have a role, but such triggers are unlikely to be associated with birth weight across three generations.

Thirdly, the intrauterine environment may be important. Mothers who have gestational diabetes are more likely to have large babies who are at risk of later type 2 diabetes. That the effect of intrauterine exposure to diabetes is not simply attributable to confounding by genetic factors has been demonstrated in Pima Indians. ${ }^{29}$ Among siblings discordant for exposure to diabetes in utero, there was an almost fourfold risk of developing diabetes in the exposed sibling compared with the sibling born before diagnosis of maternal diabetes, and therefore unexposed. No difference in risk, however, was observed between offspring born before and after the diagnosis of paternal diabetes. The daughters of rats with streptozocin induced diabetes had higher blood glucose concentrations during late pregnancy than those of control pregnant rats. ${ }^{30}$ When the mother was mildly diabetic there was fetal islet cell hyperplasia and hyperinsulinaemia with subsequent neonatal macrosomia, an effect that persisted into third generations fetuses. In this study we do not have

Table 3 Association between birth weight in mothers (F1) and type 2 diabetes in grandparents (F0)

\begin{tabular}{|c|c|c|c|}
\hline Birth weight tertile of mother (range(g)) & Number cases/non-cases & OR $(95 \% \mathrm{Cl})^{*}$ & OR $(95 \% \mathrm{Cl}) \dagger$ \\
\hline \multicolumn{4}{|l|}{ Maternal grandmother } \\
\hline $1(<3068.2)$ & $36 / 2029$ & 1 & 1 \\
\hline $2(3068.2$ to 3522.7$)$ & $25 / 1912$ & $0.72(0.43$ to 1.21$)$ & $0.72(0.43$ to 1.21$)$ \\
\hline $3(3522.8$ to 5454.5$)$ & $51 / 1814$ & 1.52 (0.98 to 2.34$)$ & $1.52(0.98$ to 2.34$)$ \\
\hline $\mathrm{p}$ for linear trend & & 0.046 & 0.046 \\
\hline ( $p$ for non-linear trend) & & $(0.035)$ & $(0.036)$ \\
\hline \multicolumn{4}{|l|}{ Maternal grandfather } \\
\hline $1(<3068.2)$ & $44 / 1717$ & 1 & 1 \\
\hline 2 (3068.2 to 3522.7$)$ & $44 / 1605$ & $1.05(0.69$ to 1.61$)$ & $1.06(0.70$ to 1.63$)$ \\
\hline $3(3522.8$ to 5454.5$)$ & $47 / 1591$ & $1.06(0.70$ to 1.61$)$ & $1.08(0.71$ to 1.64$)$ \\
\hline $\mathrm{p}$ for linear trend & & 0.79 & 0.74 \\
\hline ( $p$ for non-linear trend) & & (0.67) & (0.68) \\
\hline
\end{tabular}


Table 4 Association between diabetic status in grandparents (FO) and birth weight of grandchildren (F2)

\begin{tabular}{ll}
\hline Grandparental diabetic status ( $\mathbf{n})$ & *Adjusted mean birth weight $(95 \% \mathrm{Cl})$ \\
\hline At least one maternal grandparent (498) & $3515(3489$ to 3541$)$ \\
At least one paternal grandparent (195) & $3465(3433$ to 3498$)$ \\
No grandparents (7300) & $3438(3432$ to 3444$)$ \\
At least one maternal and one paternal grandparent (24) & $3505(3390$ to 3620) \\
\hline *Birth weight to nearest gram adjusted for sex of baby and gestational age
\end{tabular}

data on grandmaternal glucose levels in pregnancy, but the associations reported are consistent with the effect of grandmaternal increased glucose during pregnancy-that is, an excess of high birthweight babies among the offspring of the grandmothers who developed type 2 diabetes.

Finally, genetic factors may operate. Before this study, four other studies in humans have provided evidence for the intergenerational transmission of risk of low birth weight from parents with type 2 diabetes. ${ }^{17-20}$ (Studies carried out in the mid-20th century that found offspring of diabetic men to have higher birth weight than controls would have been dominated by men with type 1 diabetes, ${ }^{31}{ }^{32}$ and within individuals, higher own birth weight predicts a higher risk of developing type I diabetes ${ }^{33}$ ). In Pima Indians, a population with a high prevalence of type 2 diabetes, an inverse association between offspring birth weight and paternal diabetes has been described, ${ }^{17}$ but birth weight was positively associated with maternal diabetes, leading the authors to suggest that the high prevalence of gestational diabetes and the large effect of maternal hyperglycaemia in this population could mask any effect of maternal genes that may also be associated with low birth weight. Recently this possibility has been confirmed. In a UK study, birth weight of offspring was inversely related to maternal insulin resistance in late adulthood, ${ }^{18}$ a large Swedish study found that birth weight adjusted for gestational age was inversely related to both maternal and paternal diabetic mortality, ${ }^{19}$ and in the 1958 UK birth cohort type 2 diabetes in fathers was associated with lower offspring birth weight, while a similar association with diabetic mothers was restricted to those women who were likely to have been free of metabolic disturbances related to diabetes at the time of birth. ${ }^{20}$ These studies therefore point to the role of both maternal and paternal genetic effects and offer support for the "fetal insulin hypothesis", which states that common, as yet undefined, polygenic genetic factors that increase insulin resistance, both in utero and in adult life would result in a small, thin baby and an adult with insulin resistance and increased risk of type 2 diabetes, particularly in the presence of obesity. ${ }^{7}$

Taking the intra-uterine environment and diabetic genes mechanisms together a $U$ shaped association between grandmaternal type 2 diabetes and birth weight of their offspring might be expected, with higher prevalence of grandmaternal diabetes among the women who had low and high offspring birth weight. This is indeed what is seen in this study. The associations of paternal grandparental diabetes and birth weight of grandchildren offers only weak support for the role of paternal diabetes genes, however. The hypothesis that epigenetic factors may influence diabetic risk in subsequent generations was advanced in a recent study from Sweden that found increased diabetic mortality in the grandchildren of paternal grandparents who were exposed to a surfeit of food in the growth period prior to puberty. ${ }^{34}$ Our finding that maternal grandparents of babies in the highest birthweight tertile had an increased risk of type 2 diabetes is consistent with this explanation. Scientific consideration of

Table 5 Association between birth weight in offspring (F2) and type 2 diabetes in grandparents (FO)

\begin{tabular}{|c|c|c|c|}
\hline Birth weight tertile of baby (range (g)) & Number cases/non-cases & $O R^{*}(95 \% \mathrm{Cl})$ & $\mathrm{OR}+(95 \% \mathrm{Cl})$ \\
\hline \multicolumn{4}{|l|}{ Maternal grandmother } \\
\hline $1(\leqslant 3240)$ & $48 / 2669$ & 1 & 1 \\
\hline $2(3241-3640)$ & $42 / 2821$ & 1.00 (0.65 to 1.53 ) & $1.02(0.68$ to 1.55$)$ \\
\hline $3(3641-5640)$ & $71 / 2827$ & $1.77(1.22$ to 2.59$)$ & 1.59 (1.09 to 2.33 ) \\
\hline$p$ for linear trend & & 0.001 & $<0.001$ \\
\hline (non-linear) & & $(0.84)$ & $(0.54)$ \\
\hline \multicolumn{4}{|l|}{ Maternal grandfather } \\
\hline $1(\leqslant 3240)$ & $54 / 2247$ & 1 & 1 \\
\hline $2(3241-3640)$ & $70 / 2414$ & $1.03(0.72$ to 1.50$)$ & $1.03(0.71$ to 1.48$)$ \\
\hline $3(3641-5640)$ & $83 / 2422$ & $1.56(1.11$ to 2.18$)$ & 1.59 (1.13 to 2.22$)$ \\
\hline $\mathrm{p}$ for linear trend & & 0.004 & 0.005 \\
\hline (non-linear) & & (0.58) & $(0.18)$ \\
\hline \multicolumn{4}{|l|}{ Paternal grandmother } \\
\hline $1(\leqslant 3240)$ & $34 / 1709$ & 1 & 1 \\
\hline $2(3241-3640)$ & $28 / 1741$ & 1.03 (0.64 to 1.68 ) & 1.00 (0.62 to 1.63 ) \\
\hline $3(3641-5640)$ & $27 / 1780$ & $0.67(0.39$ to 1.15$)$ & 0.65 (0.38 to 1.12 ) \\
\hline $\mathrm{p}$ for linear trend & & 0.085 & 0.13 \\
\hline (non-linear) & & $(0.036)$ & $(0.01)$ \\
\hline \multicolumn{4}{|l|}{ Paternal grandfather } \\
\hline $1(\leqslant 3240)$ & $41 / 1400$ & 1 & 1 \\
\hline $2(3241-3640)$ & $45 / 1375$ & 1.32 (0.86 to 2.03 ) & 1.50 (0.97 to 2.34$)$ \\
\hline $3(3641-5640)$ & $31 / 1438$ & $0.76(0.46$ to 1.23$)$ & $0.92(0.57$ to 1.51$)$ \\
\hline $\mathrm{p}$ for linear trend & & 0.27 & 0.77 \\
\hline (non-linear) & & $(0.026)$ & $(0.16)$ \\
\hline
\end{tabular}

*Adjusted for sex and gestational age; tadjusted for sex, gestational age, square of gestational age, height of mother, smoking status of mother in mid-pregnancy, parity, ethnicity, and additionally for age of grandparent, and smoking in that relative. 
epigenetic inheritance, until recently a neglected and somewhat maligned concept, has been advocated, ${ }^{35}$ but rigorous evaluation of this mechanism in other studies is required.

Maternal genetic effects on glucose tolerance and offspring birth weight have both been explicitly demonstrated elsewhere. ${ }^{36}{ }^{37}$ A study of mothers and infants with mutations in the gene that codes for glucokinase, suggested that a mutation in the mother resulted in an increase in fetal size, mediated through increased fetal insulin secretion in response to maternal hyperglycaemia, but the same mutation in the fetus resulted in small fetal size, mediated through decreased insulin secretion. When both mother and fetus had the glucokinase mutation, the two opposing effects cancelled out and the baby was of normal weight. ${ }^{36}$ Similar findings have been reported in mice. ${ }^{37}$ In this study the $U$ shaped association between diabetes in maternal grandmothers and their daughter's birth weight and the positive associations between diabetes in both maternal grandparents and grandchild's birth weight offer support for both intrauterine prenatal developmental and genetic effects. Exactly which genetic factors may be responsible for transmission of diabetes risk at a population level are as yet unknown.

In conclusion, the findings in this study provide, for the first time, evidence that intrauterine factors and genetic influences may contribute to the intergenerational association between risk of type 2 diabetes and birth weight in a population without a very high prevalence of type 2 diabetes. The limitations of this study point to the need to replicate the findings in studies with detailed data on growth in the first years of life together with mitochondrial and genomic DNA data on mothers, fathers, and offspring. Such investigations will improve understanding of the aetiology of this complex and increasingly prevalent disorder.

\section{ACKNOWLEDGEMENTS}

We are extremely grateful to all the mothers who took part and to the midwives for their cooperation and help in recruitment. The whole ALSPAC study team comprises interviewers, computer technicians, laboratory technicians, clerical workers, research scientists, volunteers, and managers who continue to make the study possible. The ALSPAC study is part of the WHO initiated European longitudinal study of pregnancy and childhood.

\section{Authors' affiliations \\ P McCarron, Northern Ireland Cancer Registry, Department of}

Epidemiology and Public Health, Queen's University Belfast, Belfast, UK G Davey Smith, Department of Social Medicine, University of Bristol, Bristol, UK

A T Hattersley, Department of Vascular Medicine and Diabetes Research, Postgraduate Medical School, University of Exeter, Exeter, UK ALSPAC Study Team, Unit of Paediatric and Perinatal Epidemiology, Institute of Child Health, University of Bristol, Bristol, UK

Funding: this study could not have been undertaken without the financial support of the Medical Research Council, the Wellcome Trust, various government departments, medical charities, and commercial companies. PMcC was funded by a Fellowship in Cancer Epidemiology from the Research and Development Office for the Health and Personal Social Services, Northern Ireland.

Conflicts of interest: none declared.

\section{REFERENCES}

1 McCance DR, Pettitt DJ, Hanson RL, et al. Birth weight and non-insulin dependent diabetes: thrifty genotype, thrifty phenotype, or surviving small baby genotype? BMJ 1994;308:942-5.

2 Hales CN, Barker DJ, Clark PM, et al. Fetal and infant growth and impaired glucose tolerance at age 64. BMJ 1991;303:1019-22.

3 Rich-Edwards JW, Colditz GA, Stampfer MJ, et al. Birthweight and the risk for type 2 diabetes mellitus in adult women. Ann Intern Med 1999;130:278-84.
4 Silverman BL, Rizzo TA, Cho NH, et al. Long-term effects of the intrauterine environment. The Northwestern University Diabetes in Pregnancy Center. Diabetes Care 1998;21(suppl 2):B142-9.

5 Hales CN. The pathogenesis of NIDDM. Diabetologia 1994;37(suppl 2): S162-8.

6 Barker DJ. Intrauterine programming of adult disease. Mol Med Today 1995; 1:418-23.

7 Hattersley AT, Tooke JE. The fetal insulin hypothesis: an alternative explanation of the association of low birthweight with diabetes and vascular disease. Lancet 1999;353:1789-92.

8 Emanuel I, Filakti H, Alberman E, et al. Intergenerational studies of human birthweight from the 1958 birth cohort. II. Do parents who were twins have babies as heavy as those born to singletons? Br J Obstet Gynaecol 1992;99:836-40.

9 Little RE. Mother's and father's birthweight as predictors of infant birthweight. Paediatr Perinat Epidemiol 1987;1:19-31.

10 Ounsted M, Scott A, Ounsted C. Transmission through the female line of a mechanism constraining human fetal growth. Ann Hum Biol 1986;13:143-51

11 Godfrey KM, Barker DJ, Robinson S, et al. Maternal birthweight and diet in pregnancy in relation to the infant's thinness at birth. Br J Obstet Gynaecol 1997; 104:663-7.

12 Stewart RJ, Sheppard H, Preece R, et al. The effect of rehabilitation at different stages of development of rats marginally malnourished for ten to twelve generations. Br J Nutr 1980;43:403-12.

13 Davey Smith G, Hart C, Ferrell C, et al. Birth weight of offspring and mortality in the Renfrew and Paisley study: prospective observational study. BMJ 1997;315:1189-93.

14 Davey Smith G, Harding S, Rosato M. Relation between infants' birth weight and mothers' mortality: prospective observational study. BMJ 2000;320:839-40.

15 Davey Smith G, Whitley E, Gissler M, et al. Birth dimensions of offspring, premature birth, and the mortality of mothers. Lancet 2000;356:2066-7.

16 Barker DJP. Fetal programming and public health. In: O'Brien PMS, Wheeler T, Barker DJP, eds. Fetal programming: Influences on development and disease in later life. London: RCOG Press, 1999:3-11.

17 Lindsay RS, Dabelea D, Roumain J, et al. Type 2 diabetes and low birth weight: the role of paternal inheritance in the association of low birth weight and diabetes. Diabetes 2000;49:445-9.

18 Lawlor DA, Davey Smith G, Ebrahim S. Birth weight of offspring and insulin resistance in late adulthood: cross sectional survey. BMJ 2002;325:359.

19 Davey Smith G, Sterne JAC, Tynelius P, et al. Birth characteristics of offspring and parental diabetes: evidence for the fetal insulin hypothesis. J Epidemiol Community Health 2004:58:126-8.

20 Hypponen E, Davey Smith G, Power C. Parental diabetes and birth weight of offspring: intergenerational cohort study. BMJ 2003;326:19-20.

21 Golding J, Pembrey M, Jones R. ALSPAC: the Avon longitudinal study of parents and children. I. Study methodology. Paediatr Perinat Epidemiol 2001;15:74-87.

22 ALSPAC. Children of the nineties. (http://www.alspac.bristol.ac.uk/).

23 Gatling W, Hill RD. General characteristics of a community-based diabetic population. Practical Diabetes 1988;5:104-7.

24 McKeigue PM, Pierpoint P, Ferrie JE, et al. Relationship of glucose intolerance and hyperinsulinaemia to body fat pattern in South Asian and Europeans. Diabetologia 1992;35:785-91.

25 Ismail AA. The burden of diabetes in north Liverpool: epidemiology, complications and coronary artery disease risk factors. [PhD thesis]. Liverpool: University of Liverpool, 1998.

26 Vanderpump MP, Tunbridge WM, French JM. The incidence of diabetes mellitus in an English community: a 20-year follow-up of the Whickham survey. Diabet Med 1996;13:741-6.

27 Gayle HD, Yip R, Frank MJ, et al. Validation of maternally reported birth weights among 46,637 Tennessee WIC program participants. Public Health Rep 1988;103:143-7.

28 Wilcox WD, Gold BD, Tuboku-Metzger AJ. Maternal recall of infant birth weight. Clin Pediatr (Phila) 1991;30:509-10.

29 Dabelea D, Hanson RL, Lindsay RS, et al. Intrauterine exposure to diabetes conveys risks for type 2 diabetes and obesity: a study of discordant sibships. Diabetes 2000;49:2208-11

30 Aerts L, Holemans K, Van Assche FA. Maternal diabetes during pregnancy: consequences for the offspring. Diabetes Metab Rev 1990;6:147-67.

31 Jackson WPU. A concept of diabetes. Lancet 1955;ii:625-31.

32 Kellock TD. Birthweight of children of diabetic fathers. Lancet 1961;i:1252-4.

33 Stene LC, Magnus P, Lie RT, et al. Norwegian childhood Diabetes Study Group. Birth weight and childhood onset type 1 diabetes: population based cohort study, BMJ 2001;322:889-92.

34 Kaati G, Bygren LO, Edvinsson S. Cardiovascular and diabetes mortality determined by nutrition during parents' and grandparents' slow growth period. Eur J Hum Genet 2002;10:682-8.

35 Pembrey ME. Time to take epigenetic inheritance seriously. Eur J Hum Genet 2002;10:669-71.

36 Hattersley AT, Beards F, Ballantyne E, et al. Mutations in the glucokinase gene of the fetus result in reduced birth weight. Nat Genet 1998;19:268-70.

37 Terauchi $\mathrm{Y}$, Kubota N, Tamemoto H, et al. Insulin effect during embryogenesis determines fetal growth: a possible molecular link between birth weight and susceptibility to type 2 diabetes. Diabetes 2000;49:82-6. 